\title{
The Relationship Between the Sensor Networks Performance and the Number of Reporting Nodes
}

\author{
Fatma Othman and Nizar Bouabdallah \\ INRIA-IRISA, Campus de Beaulieu 35042 Rennes France. \\ \{fatma.othman,nizar.bouabdallah\}@inria.fr
}

\begin{abstract}
Energy-efficiency is one of the major concerns in wireless sensor networks since it impacts the network lifetime. In this paper, we investigate the relationship between sensor networks performance, particularly its lifetime, and the number of reporting nodes $N$ by using both analytical and simulation approaches. We first show that the network lifetime and the number of correctly received reports increase when $N$ decreases. Moreover, we demonstrate that the average time required to report an event is a convex function of $N$. Based on these results, and as a main contribution, we prove that the optimal number of reporting nodes minimizing the energy consumption in the network does not correspond to the optimal number of reporting nodes allowing the fastest way to report an event. The tradeoff between these two requirements is therefore specific to each sensor application, depending on its particular needs. In this paper, we provide a simple methodology to achieve this tradeoff.
\end{abstract}

\section{INTRODUCTION}

Energy-efficiency is a critical issue in wireless sensor networks (WSNs) due to the limited capacity of the sensor nodes' batteries. Indeed, once a WSN is in place, its lifetime must last as long as possible based on the initially provided amount of energy. In view of this, techniques minimizing energyconsumption are required to improve the network lifetime. A frequently used mechanism is to schedule sensor nodes activity so that redundant nodes enter the sleep mode as often as possible [1] [2] [3]. Another solution to reduce energy consumption, consists on realizing congestion control in order to avoid energy wastage due to frequently occurring collisions in such WSN networks [4].

The majority of previous works focus only on the energy minimization problems. Whereas, minimizing the energy consumption must be achieved while respecting the specific QoS requirements of sensor applications, such as the maximum tolerable time to report an event, and the required event reliability, etc. Indeed, the key behavior metrics in WSN networks are both the network lifetime and the average time required to report reliably an event. The optimal solution must therefore combine these two metrics. In view of this, in this paper, we study the tradeoff between the above mentioned metrics.

Moreover, previous works handled the energy optimization issue without paying attention to the impact of the number of reporting nodes on the network lifetime. In other words, given a reporting frequency, how the network lifetime evolves with respect to the number of active reporting nodes. To the best of our knowledge, we are the first to tackle the energy optimization problem from this perspective.

In this paper, we explore the relationship between the WSN performances (i.e. network lifetime, event reporting time) and the number of active reporting nodes, given a predefined network reporting frequency. To achieve this, we analyze the basic access mechanism IEEE 802.11 DCF (distributed coordination function) with its optional request-to-send/clearto-send (RTS/CTS) scheme. This protocol is used by the sensor nodes to arbitrate their access to the common bandwidth in order to communicate with the sink node. We derive the expression of the collision probability as a function of the number of reporting nodes and the reporting frequency. Based on these results, and as a first main contribution of this paper, we prove, by means of simulations, that the network lifetime increases when decreasing the number of active reporting nodes. Simulation results show that the maximal network lifetime is achieved when only one reporting node is activated while the remaining nodes undergo the sleep mode. Indeed, in doing so, collisions among reporting nodes is avoided, eliminating thus unnecessary energy consumption. On the other side, simulation results illustrate that the time required to report an event is a convex function of the number of active reporting nodes $N$, where the minimum is obtained for $N_{\text {opt }}>1$. Consequently, we demonstrate that the fastest way to report reliably an event does not really lead to the most efficient energy consumption. The tradeoff between these two requirements (i.e. energy consumption and reporting time) depends mainly on the specific sensor application needs.

The remainder of the paper is organized as follows. Section II presents the general problem statement. A brief description of the used MAC protocol is outlined in section III. In section IV, we introduce a mathematical model to evaluate the impact of the number of reporting nodes and the reporting frequency on the collision probability in WSNs. Analytical and simulation results are discussed in section V. In section VI, we provide a simple methodology to achieve the tradeoff between energy consumption and reporting latency. Finally, section VII concludes this paper.

\section{Problem Statement}

Let us consider the WSN as depicted in Fig. 1. In essence, a WSN ensures the supervision of a given area by the use of a sink node, which collects reports from the network. In this 


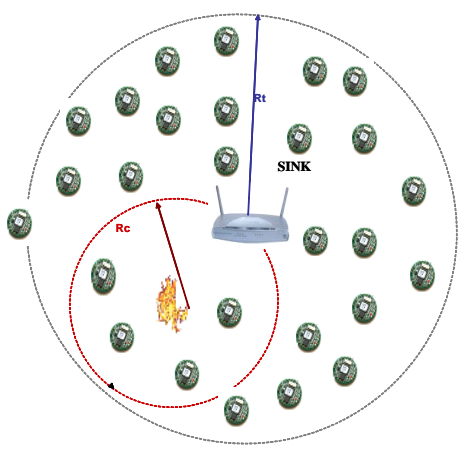

Fig. 1. Example of a sensor network.

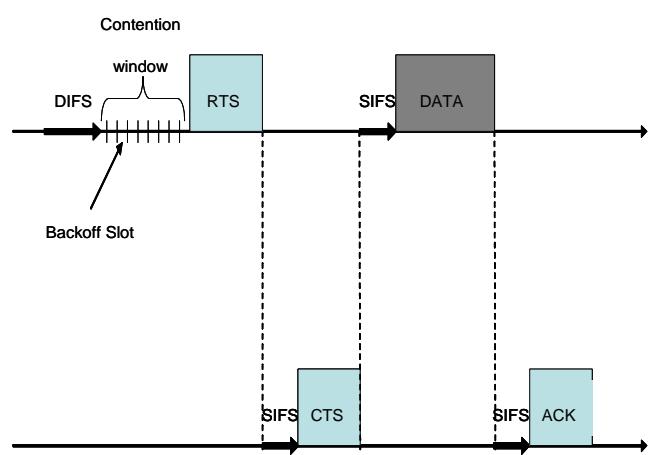

Fig. 2. Basic access mechanism of IEEE 802.11 DCF. analysis we consider event detection driven wireless sensor applications. In other words, communications are triggered by the occurrence of a pre-specified type of events. Once an event occurs, it has to be reported to the sink by the sensor nodes. In such network, sensor nodes, within an event radius $R_{c}$, are the sources (i.e. reporting nodes) for the detected event (see Fig. 1). Recall that sensor nodes are characterized by their coverage range $R_{c}$ and transmission range $R_{t}$. For the sake of simplicity, in this work, as in [4], we suppose that all the sensor nodes are within one hop from the sink.

Henceforth, we denote by $N$ the number of reporting nodes for a detected event. Moreover, we denote by $f$ the network reporting frequency. The network reporting frequency is defined as the number of packets generated per unit of time by the network to report an event. Hence, given $N$ reporting nodes, the reporting frequency of each sensor node must be set equal to $f_{s}=f / N$ to get the predefined network reporting frequency. This parameter $f$ is generally fixed by the network administrator in order to achieve required event detection reliability, $R$. The desired event reliability, $R$, is the number of data packets required by the sink to consider the event as reliable. Once the sink node receives $R$ reports, it asks the sensor nodes to stop the event reporting.

In this study, we aim at analyzing the impact of the number of reporting nodes $N$ on the WSN performance. The basic idea behind our proposal is to let some potential reporting nodes to enter a sleep mode. In the extreme case, we only let one sensor node $(N=1)$ to report a detected event with a reporting frequency $f_{s}=f$. Furthermore, we evaluate the collision probability, the average time required to report an event and the network lifetime as a function of the number of active reporting nodes $N$.

\section{Wireless SENSOR NeTWORKS}

As stated before, communications in WSN are carried using the basic IEEE 802.11 DCF protocol and its optional RTS/CTS mechanism. Specifically, once an event is detected, the $N$ active reporting nodes compete to access the common data channel to report the event to the sink. The IEEE 802.11 DCF access method is based on the CSMA/CA technique. Accordingly, a host, wishing to transmit a frame, first senses the channel activity until an idle period equal to Distributed Inter Frame Space (DIFS) is detected. Then, the station waits for a random backoff interval before transmitting. The backoff time counter is decremented in term of time slots as long as the channel is sensed free. The counter is suspended once a transmission is detected on the channel. It resumes with the old remaining backoff interval when the channel is sensed idle again for a DIFS period. The station transmits its frame when the backoff time becomes zero. In this case, the host starts the process by sending a RTS frame.

If the frame is correctly received, the receiving host sends a CTS frame after a Short Inter Frame Space (SIFS). Once the CTS frame is received, the sending host transmits its data frame. If the sending host does not receive the CTS frame, a collision is assumed to have occurred. In this case, the sending host attempts to send the RTS frame again when the channel is free for a DIFS period augmented by the new backoff, which is calculated as follows.

For each new transmission attempt, the backoff interval is uniformly chosen from the range $[0, C W]$ in term of slot of times. At the first transmission attempt of a frame, $C W$ equals the initial backoff window size $C W_{\min }=31$. Following to each unsuccessful transmission, $C W$ is doubled until a maximum backoff windows size value $C W_{\max }=1023$ is reached. Once the frame is successfully transmitted, the $C W$ value is reset to $C W_{\min }$. Figure 2 illustrates the IEEE 802.11 DCF access mechanism.

\section{Performance Analysis}

In this section, we present a mathematical model to derive collision probability as a function of the number of reporting nodes $N$ and the reporting frequency $f$. We note that collision, which occurs due to multiple reporting nodes' access, is a key factor that impacts the total energy consumption as well as the time required to report an event. In fact, the more frequent the collisions are, the more time and energy are spent to report an event.

In this study, we distinguish between two modes of functioning according to the network reporting frequency $f$ : the saturated and unsaturated regimes. The first mode is obtained when $f$ is high enough. In this case, each time the channel 
is free for transmission, each station among the $N$ reporting ones has at least one report to transmit. In other words, for each new transmission cycle, all the reporting nodes compete to access the common channel. In contrast, in the unsaturated regime, it may happen that the channel remains free. This is the case if $f$ is chosen to be relatively low.

\section{A. Probability of collision in the saturated regime}

Assume $N$ reporting stations contending to access the common channel. In saturation conditions, each station has always a report to transmit. In this case, a collision occurs when two or more backoff counters $B_{i}(i=1, \ldots, N)$ of different stations expire at the same time.

Hereafter, we assume that the number of transmissions that are subject to multiple successive collisions is negligible. This assumption is widely used in literature to simplify the analytical models. Accordingly, following to a successful transmission, we can also assume that the backoff $B_{i}(i=$ $1, \ldots, N)$ of each reporting station takes a value in $\left[0, C W_{\min }\right]$. This second assumption holds since we omit successive collisions occurrence as explained in [5]. The accuracy of these approximations is justified, as it will be demonstrated in the next section, through the perfect match between the analytical and simulation results.

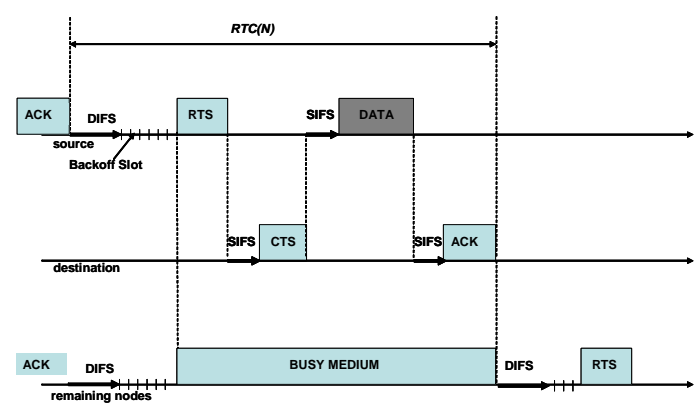

(a) Successful transmission from the first attempt

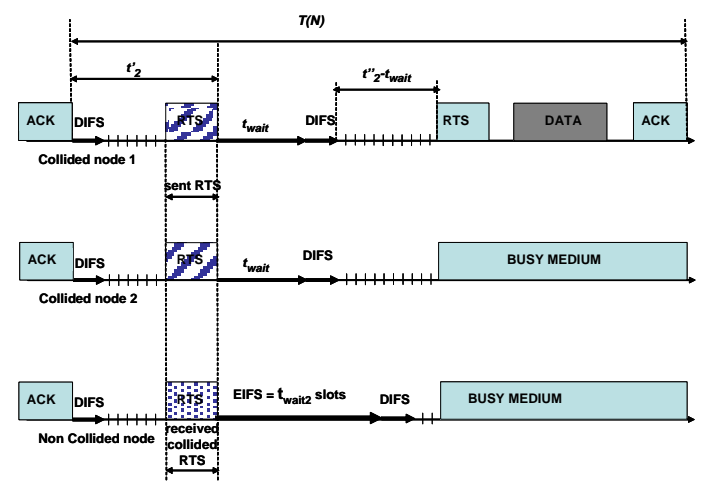

(b) Successful transmission after a first failed attempt

Fig. 3. The reporting transmission cycle RTC.

Let us now calculate the probability of collision occur- rence $P_{\text {col sat }}(N)$ when reporting an event, that is during a reporting transmission cycle (RTC). The RTC is defined as the time spent between two successive acknowledgment transmissions by the sink node. Recall that the sink node sends an acknowledgment frame after the reception of each report. In other words, RTC is the time required by the WSN to report an event to the sink. Before we delve in calculations, as a first main contribution, we outline that our method gives simple expression and more accurate results of the collision probability than [5].

As we neglect multiple successive collisions occurrence, during an RTC cycle, a report can be either successfully transmitted from the first attempt (Fig. 3.a), or following to a first collision (Fig. 3.b). Hence, a collision can only occur at the beginning of the RTC cycle with a probability $P_{\text {col sat }}(N)=P_{c}(N)$, where $P_{c}(N)$ is the probability of collision among $N$ competing access nodes with their associated backoffs $B_{i}(i=1, \ldots, N)$ ranging between $\left[0, C W_{\text {min }}\right]$.

A collision occurs when several backoff counters expire at the same time. Hence, the probability of collision $P_{c}(N)$ can be written as follows:

$$
P_{c}(N)=\operatorname{Pr}\{\bar{U}\}=\sum_{k=0}^{C W_{\text {min }}} \operatorname{Pr}\{X=k, \bar{U}\}
$$

where the random variable $\mathrm{X}$ denotes $\left(\min _{i \in<1, N>} B_{i}\right)$ and the event $\bar{U}$ is defined as follows:

$$
\begin{aligned}
\bar{U} & =\left\{\exists i, j \in<1, N>, i \neq j, B_{i}=B_{j}=X\right\} \\
& =\{\text { Collided transmission }\} .
\end{aligned}
$$

The event $\{X=k, \bar{U}\}$ simply implies that the backoff counter becomes zero for the first time in $k$ slots for at least two stations, which leads to a collision occurrence. Thus, $\operatorname{Pr}\{X=$ $k, \bar{U}\}$ can be derived as follows:

$$
\operatorname{Pr}\{X=k, \bar{U}\}=\sum_{i=2}^{N}\left(\begin{array}{c}
N \\
i
\end{array}\right) \frac{\left(C W_{\min }-k\right)^{N-i}}{\left(C W_{\min }+1\right)^{N}}
$$

\section{B. Probability of collision in the unsaturated regime}

In the unsaturated regime, the reporting frequency of each station is relatively low. Specifically, a reporting node may have no report to transmit at the beginning of a new RTC cycle. Note that previous works limited their study to the saturated regime omitting the unsaturated one. In other words, collision probability is always calculated considering the saturated regime. So, to the best of our knowledge, and as a second main contribution of this paper, we are the first to derive this parameter in the unsaturated regime.

In order to compute the probability of collision, we assume that all the reporting nodes detect an occurring event exactly at the same time. Then, they will try to send new reports each $T=1 / f_{s}$ units of time until the desired event reliability $R$ is attained. In the unsaturated regime, we deal with successive cycles of $T$ units of time. During each cycle $N$ reports are transmitted to the sink (see Fig. 4). Each cycle of $T$ units of time is thus composed of $N$ successive RTC cycles, 


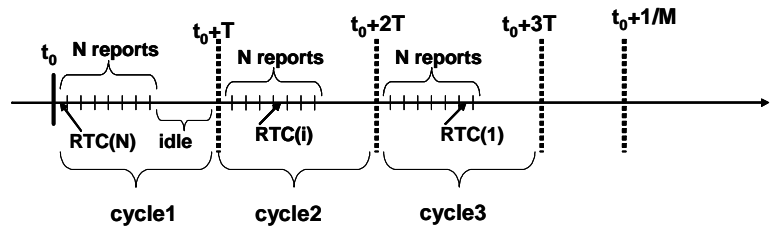

Fig. 4. Transmission cycles in the unsaturated regime.

corresponding to the $N$ reports' transmissions, followed by an idle period. This idle period is interrupted, and thus the next cycle $T$ begins, as soon as the reporting nodes generate their new reports. In this regard, $T$ can be expressed as follows:

$$
T=\sum_{i=1}^{N} R T C(i)+\text { idle_period }
$$

where $R T C(i)$ corresponds to the time required by the WSN to report an event to the sink when the number of active reporting nodes (i.e. nodes that have not yet transmitted their reports) is $i$.

Specifically, at the beginning of a cycle $T$, all the $N$ reporting nodes generate new reports to transmit to the sink. Immediately, the reporting nodes leave their idle states (as we deal with the unsaturated regime see Fig.4) and proceed according the DCF algorithm, described in section III, to transmit their reports.

Let us now calculate the collision probability among access nodes trying to report the detected event. It is defined as the probability of collision when there is at least one packet to be sent by the $N$ reporting nodes. Hence, the probability of collision can be written as follows:

$$
P_{\text {col_unsat }}(N)=\operatorname{Pr}\{\text { Collision occurs } \mid Y \geq 1\}
$$

where $Y$ denotes the stationary state of the stochastic process $\{Y(t), t \geq 0\}$, which represents the number of reporting nodes still having a packet to transmit. By conditioning on the stationary state $Y$, we get:

$$
\begin{aligned}
P_{\text {col_unsat }}(N)=\sum_{i=1}^{N} \operatorname{Pr}\{\text { Collision occurs } \mid Y=i\} \\
\times \operatorname{Pr}\{Y=i \mid Y \geq 1\}
\end{aligned}
$$

where $\operatorname{Pr}\{$ Collision occurs $\mid Y=i\}$ can be derived simply using (1) as follows:

$$
\operatorname{Pr}\{\text { Collision occurs } \mid Y=i\}=P_{c}(i)
$$

On the other side, the expression of $\operatorname{Pr}\{Y=i \mid Y \geq 1\}$, is simply given by:

$$
\operatorname{Pr}\{Y=i \mid Y \geq 1\}=\frac{R T C(i)}{\sum_{j=1}^{N} R T C(j)} \simeq \frac{1}{N}
$$

\begin{tabular}{|l|l|}
\hline Communication range & $40 \mathrm{~m}$ \\
\hline Sensing range & $30 \mathrm{~m}$ \\
\hline Packet length & 30 bytes \\
\hline IFQ length & 65 packets \\
\hline Transmit power & $0.660 \mathrm{~W}$ \\
\hline Receive power & $0.395 \mathrm{~W}$ \\
\hline Idle power & $0.035 \mathrm{~W}$ \\
\hline Initial network energy & $100 \mathrm{~J}$ \\
\hline
\end{tabular}

TABLE I

SIMULATION PARAMETERS

Finally, we derive the expression of the collision probability as follows:

$$
P_{\text {col_unsat }}(N)=\frac{1}{N} \sum_{i=1}^{N} P_{c}(i)
$$

\section{Performance Evaluation}

In this section, we analyze the impact of the reporting nodes on the WSN performances using both analytical and simulation approaches. The simulations, which are used to assess the accuracy of our analytical model, are run on NS-2 simulator.

In our simulations, we have not assumed the mobility of the sensor nodes. Therefore, the topology does not continuously vary with time during simulations. However, note that the sensor nodes may die due to energy depletion leading to variation in overall topology. Communications between sensor nodes are assured by both IEEE 802.11 DCF MAC protocol and Ad-hoc On Demand Distance Vector Routing (AODV) [6] protocol. The parameter settings in our experiments are listed in table I.

Figures 5.a and 5.b represent the probability of collision in the saturated and unsaturated regimes, respectively, for a varying number of reporting nodes. We can see that this probability increases, in both cases, with the increase of the reporting nodes. Indeed, collisions become more frequent when the number of competing access nodes increases, which leads to increasingly extra energy expenditure and increases the average time to report an event. To alleviate these issues, we have to reduce the number of reporting nodes. We note that Fig. 5 shows a quite perfect match between analytical and simulation results, which exhibits the accuracy of our models. Moreover, Fig. 5.a shows that our analytical formula match better the simulation results than the one given in [5].

Figure 6 reports the average time required by the WSN to report an event (i.e. RTC) in both saturated and unsaturated regimes. Figure 6.a shows that the RTC cycle in the saturated regime $\left(R T C_{\text {sat }}(N)\right)$ is a convex function of $N$ where the minimum is obtained for $N_{\text {opt }}=8$. In fact, the optimal RTC is a tradeoff between two opposite requirements. On the first hand, increasing the number of reporting nodes, enables a faster access to the medium during each RTC cycle since the average backoff time required by a host to successfully report an event $\left(\right.$ i.e. $\left.\min _{i=<1, N>} B_{i}\right)$ decreases when the number of reporting nodes increases. Doing so, the average RTC time 


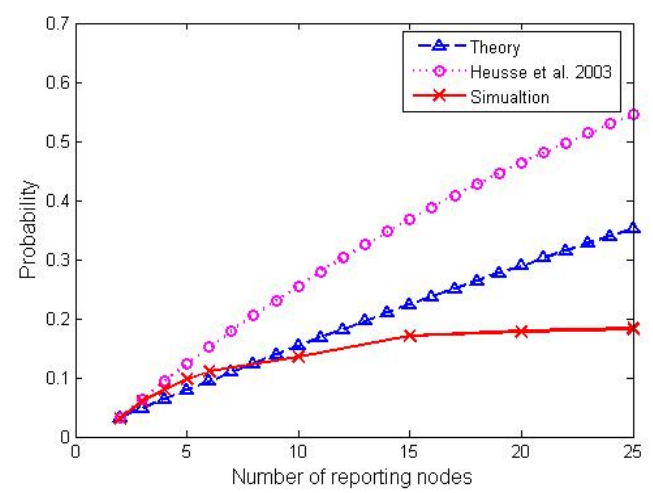

(a) The saturated regime

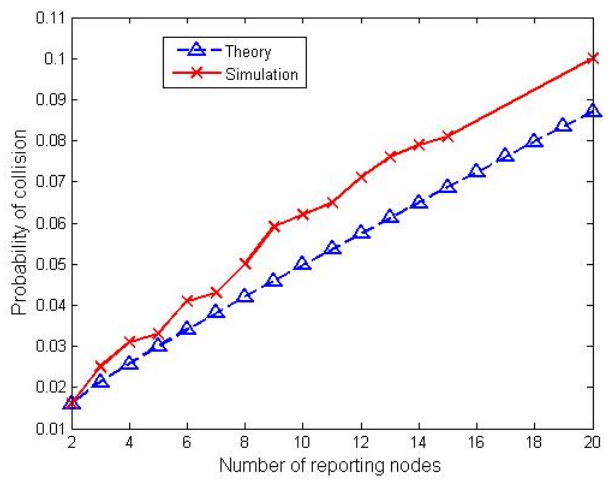

(b) The unsaturated regime

Fig. 5. Probability of collision

decreases. On the other hand, raising the number of reporting nodes, increases the probability of collision (see Fig. 5.a) which amplifies the time lost in contention procedure during each RTC cycle. Hence, reconciling the above mentioned opposite requirements, the minimum RTC time is obtained for $N_{o p t}=8$. In this regard, the fastest way to report reliably an event in the saturated regime is to devise a network where the number of active reporting nodes is set equal to $N_{o p t}$.

Similar behavior is also observed in the unsaturated regime (Fig. 6.b) where the minimum RTC time $\left(R T C_{\text {unsat }}(N)\right)$ is obtained in this case for $N_{o p t}=14$. In other words, considering a particular reporting node frequency $f$ belonging to the unsaturated range, the fastest way to report reliably an event is to let only $N_{\text {opt }}$ nodes among the $N$ potential ones to report a detected event. In this case, the remaining $\left(N-N_{\text {opt }}\right)$ reporting nodes undergo a sleep mode.

Figures 7.a and 7.b show the average amount of energy consumed during each RTC cycle (i.e. to report an event) for a varying number of reporting nodes $N$ in the saturated and unsaturated regimes, respectively. Unlike, the RTC curves, these figures show that the amounts of energy $E_{R T C_{-} s a t}(N)$ and $E_{R T C \_ \text {unsat }}(N)$ are monotonically rising with $N$. This monotonically increase is mainly due to two factors. First, increasing $N$ amplifies the wasted energy due to collisions. Moreover, increasing $N$ means waking up more sensor nodes

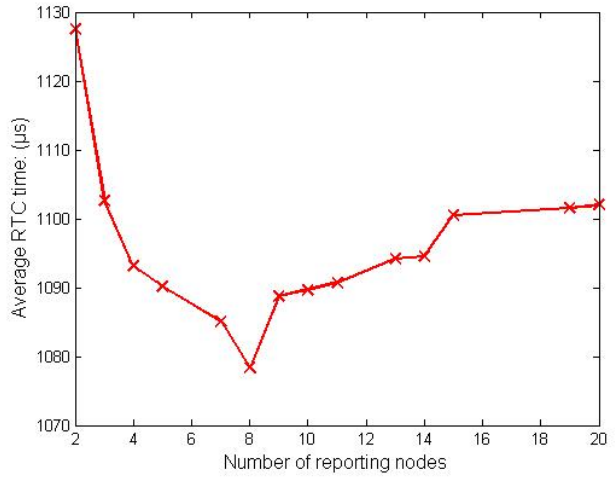

(a) The saturated regime

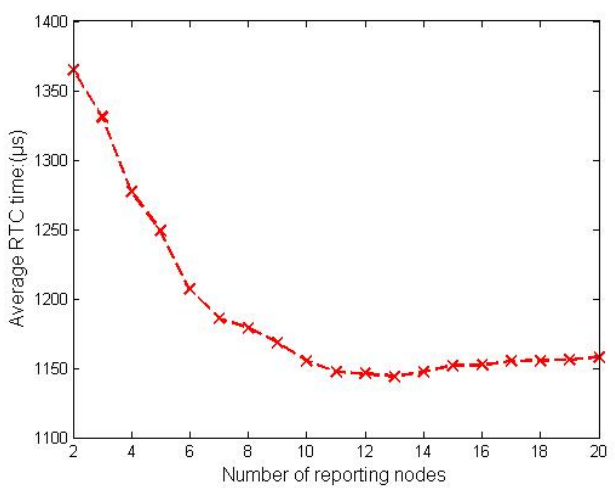

(b) The unsaturated regime

Fig. 6. Average time to report an event (RTC)

within the event radius $R_{c}$. Doing so, the total amount of energy consumed by the network in the reception of the signaling messages (RTS, ACK) increases considerably (see the hatched zones of Fig. 7). Finally, we underline that the impact of these two factors is dominant with respect to the slight idle energy reduction when $N$ increases due to the faster access to the medium. According to these results, we can see that as expected having a single reporting node consumes the least amount of energy for both saturated and unsaturated regimes. Hence, we can conclude that the fastest way to report an event does not correspond to the optimal manner to consume the network energy. In this regard, the choice of the number of active reporting nodes depends mainly on the WSN application.

Figures 8.a and 8.b plot the network lifetime evolution, in both saturated and unsaturated regimes, respectively, as a function of the number of reporting nodes $N$ for a varying values of the desired event reliability $R$. In our simulations, we consider the rate of event occurrence $M=5$. In other words, it occurs in average 5 events per unit of time. Moreover, we assume that the mean time between two successive events is higher than the mean time required to report reliably an event. Figure 8 shows that the smaller $N$ is, the longer the network lifetime becomes whatsoever the value of $R$. Thus, 


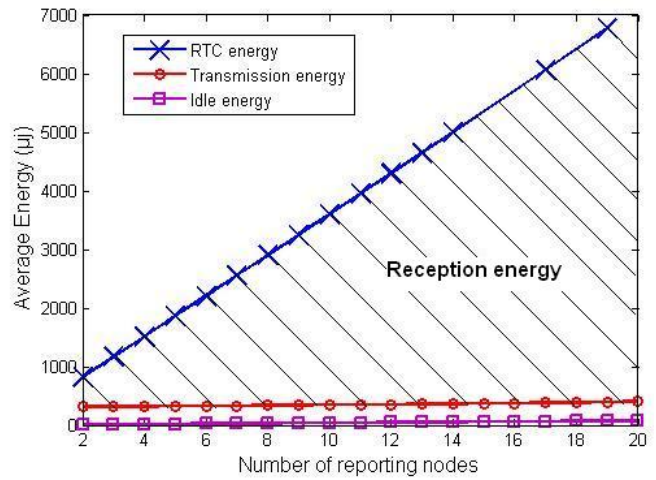

(a) The saturated regime

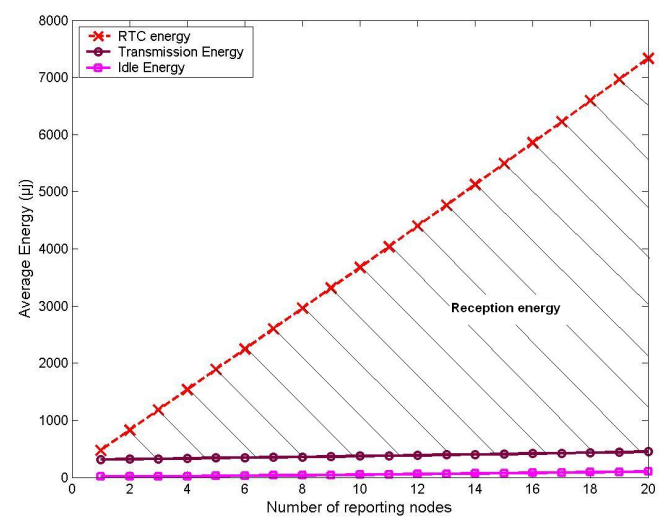

(b) The unsaturated regime

Fig. 7. The amount of enegy spent during a RTC cycle.

the maximal network lifetime is reached when $N$ is equal to 1. Indeed, when $N$ increases the probability of collision rises causing important energy depletion. In contrast, when $N$ is set equal to 1 , the probability of collision is null, avoiding thus extra energy expenditure due to collisions.

\section{TRADEOFF BETWEEN ENERGY AND LATENCY}

Energy-efficiency is a critical issue in wireless sensor networks. However, minimizing the energy consumption in such networks must be achieved while respecting the maximum tolerable time to report an event. The optimal solution must therefore take into account these two metrics.

In this section, we propose a simple function $f_{\text {choice }}$ to determine the optimal number of reporting nodes $N_{\text {opt }}$ that achieves the above-mentioned tradeoff. For illustration purposes, we give hereafter the expression of $f_{\text {choice }}$ in the saturated regime. Similar results can be easily obtained in the unsaturated regime. $f_{\text {choice }}$ can be expressed as follows:

$f_{\text {choice }}(N, \alpha)=\alpha \cdot \frac{E_{R T C_{\_} \text {sat }}(N)}{E_{\text {sat_average }}}+(1-\alpha) \cdot \frac{R T C_{\text {sat }}(N)}{R T C_{\text {sat_average }}}$

where $\alpha$ is a weight that ranges between $[0,1]$. It is fixed by the network administrator depending on the particular needs of the sensor application (i.e., whether more emphasis is given

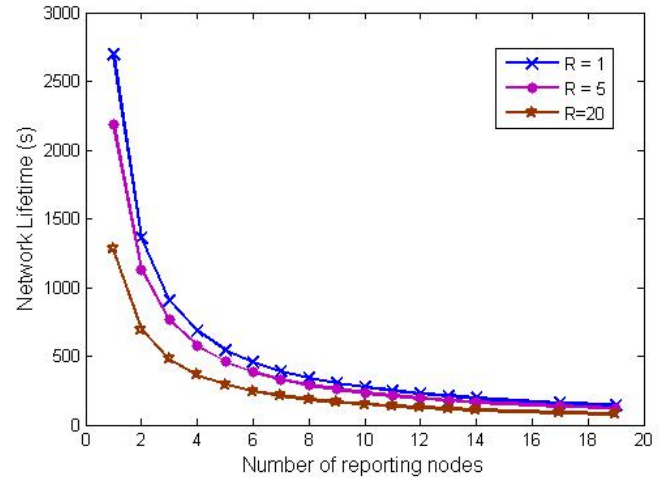

(a) The saturated regime

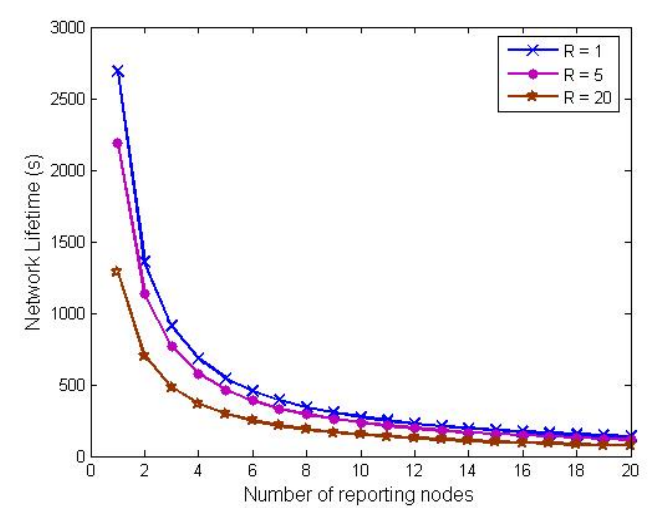

(b) The unsaturated regime

Fig. 8. Sensor Network lifetime.

to energy conservation or to reporting latency minimization), $E_{\text {sat_average }}$ and $R T C_{\text {sat_average }}$ are respectively the mean value of $E_{R T C_{\text {sat }}}(N)$ and $R T C_{\text {sat }}(N)$ for the different number of reporting nodes $N$.

Figure 9 plots the evolution of $f_{\text {choice }}$ as a function of the number of reporting nodes $N$. We consider two values of the weight $\alpha=1 / 8$ and $\alpha=1 / 4$. Two main observations can be identified through Fig. 9. First, $f_{\text {choice }}$ is a convex function of $N$ where the minimum is obtained for $N_{\text {opt }}$. In this regard, $N_{\text {opt }}$ minimizes the weighted function $f_{\text {choice }}$ and achieves therefore the desired tradeoff between the energy conservation and reporting latency minimization. Moreover, we can observe through Fig. 9 that the value of $N_{o p t}$ depends of the weight $\alpha$. Specifically, if more emphasis is given to the energy conservation aspects (i.e., $\alpha$ is set close to 1 ), the value of $N_{\text {opt }}$ will be close to $N_{\text {opt_energy }}$ (i.e., $N_{\text {opt }}=1$ ). In contrast, if more priority is given to the latency minimization aspects (i.e., $\alpha$ is set close to 0 ), the value of $N_{\text {opt }}$ will be close to $N_{\text {opt_latency }}$ (i.e., $N_{\text {opt }}=8$ ). This result is shown in Fig. 10, where $N_{o p t}$ decreases with $\alpha$ from $N_{\text {opt_latency }}$ to $N_{\text {opt_energy }}$. It is worth noting that for the extreme cases where $\alpha=0$ and $\alpha=1$ we get respectively the same curves as figs. 6.a and 7.a. 


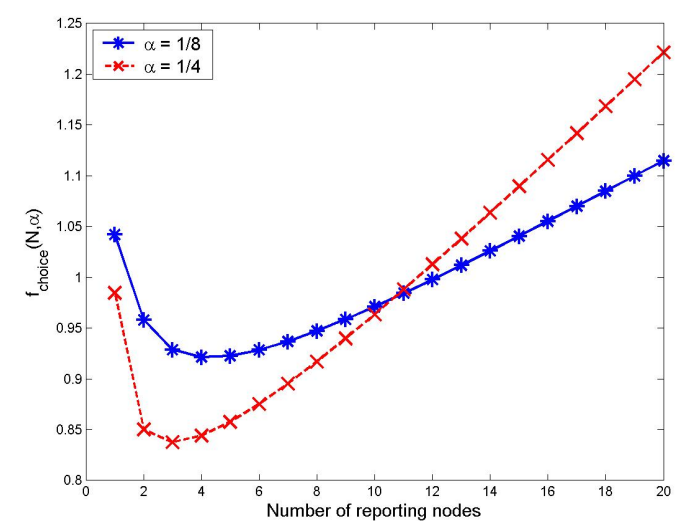

Fig. 9. Tradeoff between energy conservation and latency minimization.

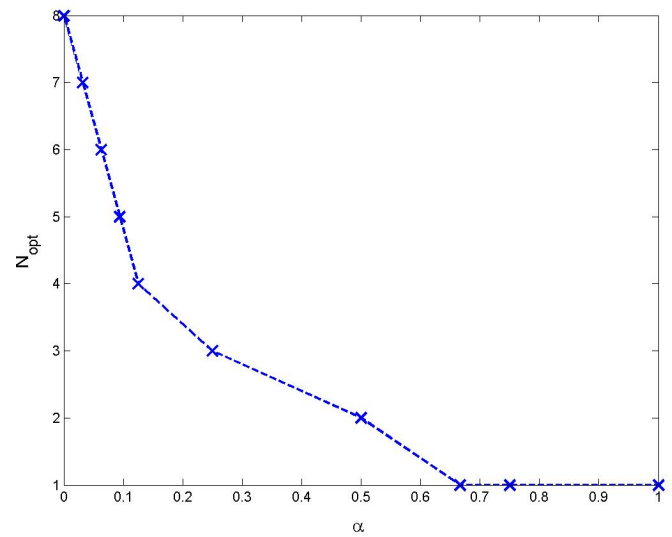

Fig. 10. The optimal number of reporting nodes for a varying value of the weight $\alpha$.

\section{CONCLUSION}

In this paper, we explored the relationship between the wireless sensor network performance and the number of reporting nodes. To the best of our knowledge, we are the first to investigate the energy optimization problem from this perspective. To achieve this, we analyzed the performance of the IEEE 802.11 MAC layer. Doing so, we first demonstrated that the optimal number of reporting nodes that minimizes the energy expenditure in the sensor network does not correspond to the fastest way to report an event. Based on this result, we propose a simple methodology to achieve this tradeoff, which depends on the specific requirements of each WSN application.

\section{REFERENCES}

[1] F. Dai and J. Wu, Distributed dominant pruning in ad hoc wireless networks, In Proceedings of IEEE International Conference on Communications, Anchorage, USA, May 2003.

[2] M. Cardei, D. MacCallum, X. Cheng, M. Min, X. Jia, D. Li and D.-Z. $\mathrm{Du}$, Wireless sensor networks with energy efficient organization, Journal of Interconnection Networks 3 (3-4) (2002) 213-229.

[3] D. Tian and N.D. Georganas, A Coverage-Preserving Node Scheduling Scheme for Large Wireless Sensor Networks, In Proceedings of the First ACM Workshop on Wireless Sensor Networks and Applications, New York, USA, 2002.
[4] O. B. Akan end I. F. Akyildiz, Event-to-Sink Reliable Transport for Wireless Sensor Networs, IEEE/ACM Transactions on Networking, vol. 13, no. 5, pp. 1003-1016, October 2005.

[5] M. Heusse, F. Rousseau, G. Berger-Sabbatel and A. Duda, Performance Anomaly of $802.11 \mathrm{~b}$, In Proceedings of IEEE INFOCOM 2003, San Francisco, USA, March 2003.

[6] S. Tilak, N. B Abu-Ghazaleh, and W. Heinzelman, Infrastructure Tradeoffs for Sensor Networks, In Proceedings of ACM WSNA 2002, pp. 49-58, Atlanta, GA, USA, September 2002. 